\title{
Lungentuberkulose erhöht das Karzinomrisiko um das Elffache
}

In der Weltbevölkerung ist jeder Dritte mit Tuberkelbakterien infiziert, jeder Zehnte erkrankt an Tuberkulose. Besonders HIV-Infizierte sind gefährdet. Wer an Tuberkulose erkrankt ist, hat auch noch ein um das Elffache erhöhtes Risiko, an Lungenkrebs zu erkranken.

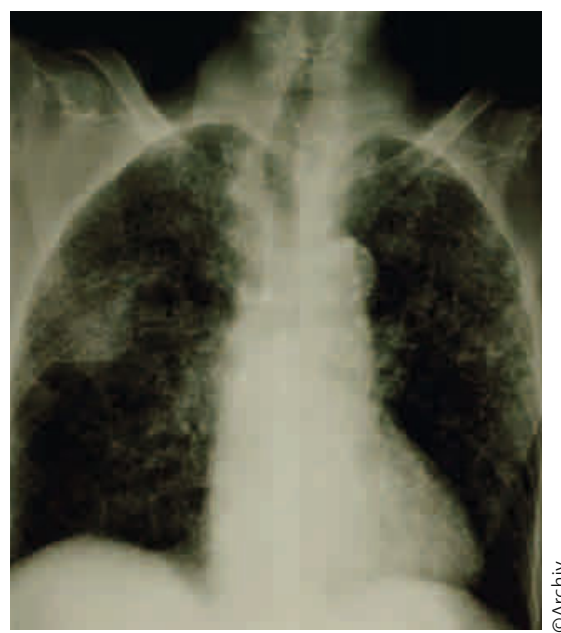

Patienten mit TBC haben ein erhöhtes Lungenkarzinomrisiko.
- Dies ergab eine große Studie von Wissenschaftlern der Medical University von Taichung (Taiwan). Dabei wurden 716872 Menschen über Jahre hinweg beobachtet. In die Studie aufgenommen wurden nur Menschen, die in ihrem Leben keine Krebsdiagnose erhalten hatten. 4480 Patienten erkrankten im Beobachtungszeitraum an Tuberkulose. Diese Gruppe wurde genutzt, um einen Zusammenhang zwischen dem Leiden und Lungenkrebs zu ermitteln. Die rund 712000 Menschen ohne Tuberkulosediagnose dienten als Kontrollgruppe.

Zwischen 2001 und 2007 ermittelten die Forscher, wie viele Studienteilnehmer an Lungenkrebs erkrankten. Dabei zeigte sich, dass das Risiko für Tuberkulosekranke rund elfmal so hoch war wie für die Menschen in der Kontrollgruppe. Während in der tuberkulosefreien Kontrollgruppe 2,4 Lungenkrebsdiagnosen pro 10000 Patientenjahre gestellt wurden, waren es in der an Tuberkulose erkrankten Gruppe
26,3. Chronisch obstruktive Lungenerkrankungen und Rauchen erhöhten das Risiko zusätzlich.

\section{-Yu,Y.-H. et al.}

(Centre for Molecular Medicine, China Medical University Hospital, 9F, No.6, Hsueh-Shih Road, Taichung 404, Taiwan; e-mail:ylyu@ mail.cmu.edu.tw) Increased lung cancer risk among patients with pulmonary tuberculosis: A population cohort study. J. Thoracic Oncol. 2011; 6: 32-37doi: 10.1097/JTO.obo13e3181fb4fcc

\section{Kommentar}

Die taiwanesischen Wissenschaftler meinen, dass ihre Erkenntnisse in die Tuberkulosebehandlung einfließen sollten - etwa in Form einer verbesserten Lungenkrebsfrüherkennung und -vorsorge für Betroffene. In Deutschland ist der größte Risikofaktor für Lungenkrebs das Zigarettenrauchen. Nach Angaben des RKI erkranken pro Jahr 50 ooo Personen an Lungenkrebs. Männer sind mehr als doppelt so oft betroffen wie Frauen.

K. MALBERG

\section{Chronische Rückenschmerzen: operieren oder nicht?}

\section{Norwegische Forscher führten eine 4-Jahres-Nachbeobachtung von 124 Patienten mit chronischen ( $>1$ Jahr) Rückenschmerzpatienten durch, die im Rahmen von zwei randomisierten Studien entweder chirurgisch oder konservativ behandelt worden waren.}

- Bei den chirurgisch behandelten Patienten wurde eine transpedikuläre $\mathrm{Fu}$ sion durchgeführt, während bei den konservativ behandelten eine kognitive Verhaltenstherapie und Physiotherapie zum Einsatz kamen. Gemessen an einer breiten Palette von Studienendpunk- ten, wie z.B. Schmerz, Funktion, Arbeitsausfall, Distress, ergaben sich keine signifikanten Gruppendifferenzen.

In vielen Variablen schnitten die konservativ behandelten Patienten sogar besser ab. Die Autoren meinen daher, dass die operative Behandlung von Rückenschmerzen der konservativen Therapie nicht überlegen sei.

\section{Kommentar}

Die Indikation zum operativen Vorgehen wird bei Rückenschmerzen häufig zu großzügig gestellt. Das wurde schon lange vermutet, wiederholt geäußert und ist durch diese Analyse erneut belegt. Wenn man zudem die Kosten und die Risiken einer Operation bedenkt, fällt das Urteil wohl noch eindeutiger aus. Das Problem ist natürlich, dass die konservative Therapie auch reichlich wenig zu bieten hat, was wirklich hilft. Aber das ist sicher kein guter Grund, zum Messer zu greifen.

E. ERNST =

\section{- J. I. Brox et al.}

Four-year follow-up of surgical versus non-surgical therapy for chronic low back pain. Ann. Rheum. Dis. 2010; doi: 10.1136/ ard.2009.108902 